\title{
A Dual Dynamic Programming Approach to the Detection of Spine Boundaries
}

\author{
Guo-Qing Wei ${ }^{1}$, JianZhong Qian ${ }^{1}$, and Helmuth Schramm ${ }^{2}$ \\ 1 Imaging Department, Siemens Corporate Research, Inc. \\ 755 College Road East, Princeton NJ 08536, USA \\ 2 Siemens AG, Medical Engineering \\ Erlangen, Germany
}

\begin{abstract}
Spine boundary is one of the key landmarks for quantifying deformities of pathological spines. In this paper, we propose a new approach to the detection of spine boundaries. We integrate two dynamic programming procedures into a single one to enable constraints between the left and right parts of the boundary to be enforced. This dual dynamic programming approach detects two boundary curves at the same time. Moreover we propose to use angular limits in a piecewise linear model as a new smoothness constraint. This leads to a computationally efficient way of introducing high order priors. Experimental results show a very robust performance of the method.
\end{abstract}

\section{Introduction}

Boundary or curve detection is a primary task in many computer vision and image processing applications. Currently, deformable models are the most popular ones used in the literature. Representative methods of this kind include the PDE (partial differential equation)-based active contour models 42, DP (dynamic programming) methods [13] and the level-set methods [5]. Since the level set methods are less flexible in incorporating prior knowledge, they will not be further pursued in this paper.

The active contour model [4] represents one major class of contour detection methods. In an active contour model, an object boundary is represented by a collection of points interacting with each other in a way to incorporate constraints from both physics and image data. Methods based on dynamic programming follows the same spirit of considerations, but use a different optimization method. Since both the active contour methods and the dynamic programming methods are based on the minimization of an energy function containing soft constraints (smoothness constraints), it is difficult, if not impossible, to explicitly control the shape of the final contour through any analytic forms for determining the smoothness weights. In addition, the dynamic programming method has a limitation that inclusion of higher order smoothness constraints will cause an exponential increase of the search space and of the computational complexity [1], limiting further the power of external control. Most distinctively, most previous 
contour detection methods do not consider the simultaneous detection of two contours while maintaining constraints among them.

In this paper, we propose a new DP-based method to curve detection. We completely drop off the soft constraints and apply instead the limit constraints. This provides not only direct control over the final outcome of the detection, but also allows the use of high order shape constraints without sacrificing computational efficiency. Under this framework, we propose a dual-DP method that can detect two curves at the same time while maintaining constraints between them. The method is applied to the problem of spine boundary detection.

\section{Dual Dynamic Programming Method}

\subsection{Problem Statement}

A spine boundary consists of two special-property curves. They are the left and right boundaries of the spine, respectively. As a first property, both boundary curves are nearly vertical. Secondly, they are nearly parallel to each other. Figure 1 (a) depicts the left and right boundaries of a spine.

Properties delineating the spine boundaries from other anatomies are intensity edges, but what makes spine boundaries different from other boundaries or edges in other image processing tasks are that there are many anatomical structures interfering with the spine boundary. For example, there are ribs connecting to the spine, and between the left and right boundaries of the spine there are anatomies such as endplates or pedicles. These make both the boundary edge discontinuous and the region between the left and right boundaries inhomogeneous.

\subsection{Piecewise Linear Model}

To represent a spine boundary we propose a piecewise linear model. Since a spine is nearly vertical, we can parameterize the spine boundary by fixed, equally spaced points in the vertical direction. This is like to cut the image plane in vertical directions by a set of equally spaced cut lines. Suppose the y-coordinates of the cutting lines are $\left\{y_{1}, y_{2}, \ldots, y_{N}\right\}$, where $\mathrm{N}$ is the number of cut lines. The left and right boundaries of the spine can be then represented by the nodal points $P_{L}=\left\{\left(x_{L, 1}, y_{1}\right),\left(x_{L, 2}, y_{2}\right), \ldots,\left(x_{L, N}, y_{N}\right)\right\}$ and $P_{R}=\left\{\left(x_{R, 1}, y_{1}\right), \quad\left(x_{R, 2}, y_{2}\right), \ldots\right.$, $\left.\left(x_{R, N}, y_{N}\right)\right\}$, respectively. Since $y_{n}$ 's are fixed and predefined, the variables which control the shape of the boundaries are the $\mathrm{x}$-coordinates $\left(x_{L, 1}, x_{L, 2}, \ldots, x_{L, N}\right)$ and $\left(x_{R, 1}, x_{R, 2}, \ldots, x_{R, N}\right)$. The spacing between the cut lines is chosen such that it reflects the shape scale of interest of the spine; we set it to approximately half of the average vertebra height. This parameter is either manually set or obtained from statistics. Figure 1 (b) shows a piecewise linear parameterization of the spine boundary of Figure 1 (a). 


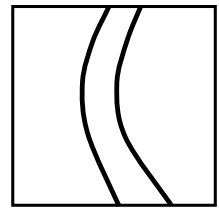

(a)

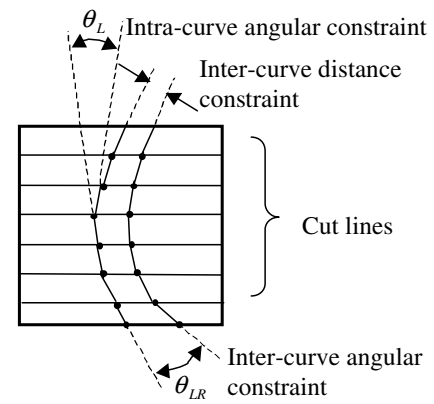

(b)

Fig. 1. The spine boundary model. (a) boundary curves; (b) piecewise linear model, with some geometric constraints illustrated.

\subsection{Geometric Constraints}

There are two kinds of shape constraints on a spine boundary: intra-curve and inter-curve. Intra-curve constraints describe shape constraints on individual boundary curves, whereas inter-curve constraints specify the correlations among them.

Under the piecewise linear model, intra-curve constraints place restrictions on a) the range of orientations of individual line segments, reflecting the fact that a spine is nearly vertical; b) the range of inter-segment angles for neighboring line segments, assuring that a smooth spine boundary is generated.

Denote the linear segments of the left and right boundaries by $S_{L}=\left\{s_{L, 1}\right.$, $\left.s_{L, 2}, \ldots, s_{L, N-1}\right\}$ and $S_{R}=\left\{s_{R, 1}, s_{R, 2}, \ldots, s_{R, N-1}\right\}$, respectively. The intra-curve constraints for the left boundary can be mathematically expressed as

$$
O_{\min }<O\left(s_{L, n}\right)<O_{\max } ; \quad n=1,2, \ldots N-1
$$

and

$$
\begin{gathered}
\angle\left(s_{L, n}, s_{L, n+1}\right)<\gamma_{1, \max } ; \quad n=1, \ldots N-2 \\
\angle\left(s_{L, n}, s_{L, n+1}\right)-\angle\left(s_{L, n-1}, s_{L, n}\right)<\gamma_{2, \max } ; \quad n=2, \ldots N-2
\end{gathered}
$$

where $O_{\min }, O_{\max }, \gamma_{1, \max }$ and $\gamma_{2, \max }$ are threshold values, symbol $O()$ denotes the orientation of a segment, and $\angle(.,$.$) the angle between two segments. The$ same constraints can be written for the right boundary.

It is worthwhile mentioning that equations (21) and (31) represent the $1^{\text {st }}$ order and $2^{\text {nd }}$ order smoothness constraints expressed in angular measurements, respectively. There are two major differences between the smoothness constraint used here and those found in the conventional contour detection methods. The first obvious difference is that smoothness is measured in angles instead of the 
derivatives of the coordinates of nodal points. The advantage is that angles are rotational invariant, easier to understand and control: With bounds on the angles, we can explicitly control the space of admissible curves. On the contrary, in the conventional methods of using smoothness constraints, there is no explicit relationship between the values of smoothness weights and any wanted shape measurement in the final detection. The second difference is that our smoothness constraints will not appear in the cost function used for finding the boundary. This allows the use of high order constraints without causing exponential increase in the computational cost, as will be shown later.

To apply inter-curve constraints, limits are put on the angles between a segment in the left boundary and neighboring segments in the right boundary. As has already been noted, this constraint steams from the fact that the left and right boundaries of a spine are almost parallel. The $1^{\text {st }}$ order inter-curve angular constraint can be written as

$$
\begin{gathered}
\angle\left(s_{L, n}, s_{R, n}\right)<\gamma_{L R, \max } \\
\angle\left(s_{L, n}, s_{R, n-1}\right)<\gamma_{L R, \max } \\
L\left(s_{L, n-1}, s_{R, n}\right)<\gamma_{L R, \max }
\end{gathered}
$$

where $\gamma_{L R \text {, max }}$ is the maximum angle allowed for local inter-curve segments.

Another inter-curve constraint specifies how vertebra width (or distances between nodal points) is allowed to change along the spine axis. Suppose $W_{n}$ and $W_{n+1}$ are the projections of the distances between corresponding nodal points onto directions orthogonal to the spine axis for the $n$-th and $(n+1)$-th cut lines, respectively. From the near-parallelism of the left and right boundaries, the inter-curve distance constraint can be written as

$$
d_{\min }<W_{n+1} / W_{n}<d_{\max }
$$

where $d_{\min }$ and $d_{\max }$ are predefined thresholds.

\subsection{Hard Constraints}

Hard constraints are external constraints specified by users as helps to the algorithm. One kind of such constraints are points on the boundary selected by users, e.g., per mouse click. The boundary curves are then required to pass through the given set of points. Suppose $Q_{L}=q_{L, i}, i=1, . ., K$ is a point set of this kind for the left boundary. Since the y-cutting lines are fixed, it is easy to determine which segment in the piecewise linear model should pass through which point. Suppose segment $s_{L, k}$ ought to pass through point $q_{L, i}$. The hard constraints for this condition can be written as

$$
d\left(q_{L, i}, s_{L, k}\right)<\delta
$$

where $d(q, s)$ denotes the distance from point $q$ to segment $s$, and $\delta$ is a tolerance threshold close to zero. Similar hard constraints can be defined for the right boundary. 


\subsection{Optimization}

Suppose $\mathrm{G}(\mathrm{x}, \mathrm{y})$ is the gradient magnitude of the spine image $\mathrm{I}(\mathrm{x}, \mathrm{y})$. The rule for finding the spine boundary is that it maximize the summed gradient magnitude along the path while satisfying all geometric and hard constraints. Formally, the segments $\left\{s_{L, n}\right\}$ and $\left\{s_{R, n}\right\}$ should maximize the following energy function

$$
\begin{gathered}
E=\sum_{n=1}^{N-1}\left(\sum_{(x, y) \in s_{L, n}} G(x, y)+\sum_{(x, y) \in s_{R, n}} G(x, y)\right) \\
\text { subject to }: \text { constraints (11) } \sim \text { (8) }
\end{gathered}
$$

Note that in the above expression, all points on a segment, instead of only the nodal points, are contributing to the energy function. (This is usually not the case with many existing contour detection methods.) But since the pixels on a segment are completely determined by the nodal points, we can make it explicit the dependency of the above expression on the nodal coordinates by defining

$$
\begin{aligned}
& S_{G}\left(x_{L, n}, x_{L, n+1}\right)=\sum_{(x, y) \in s_{L, n}} G(x, y) \\
& S_{G}\left(x_{R, n}, x_{R, n+1}\right)=\sum_{(x, y) \in s_{R, n}} G(x, y)
\end{aligned}
$$

Equation (9) can then be rewritten as

$$
\begin{aligned}
E= & S_{G}\left(x_{L, 1}, x_{L, 2}\right)+S_{G}\left(x_{R, 1}, x_{R, 2}\right)+S_{G}\left(x_{L, 2}, x_{L, 3}\right)+S_{G}\left(x_{R, 2}, x_{R, 3}\right)+\ldots \\
& +S_{G}\left(x_{L, N-1}, x_{L, N}\right)+S_{G}\left(x_{R, N-1}, x_{R, N}\right)
\end{aligned}
$$

If there were no inter-curve constraints, the maximization of $E$ in (13) could be obtained in two independent procedures, one for the left boundary, and the other for the right boundary:

$$
E=E_{L}+E_{R}
$$

where $E_{L}$ corresponds to the energy term of the left boundary:

$$
E_{L}=S_{G}\left(x_{L, 1}, x_{L, 2}\right)+S_{G}\left(x_{L, 2}, x_{L, 3}\right)+\ldots+S_{G}\left(x_{L, N-1}, x_{L, N}\right)
$$

A similar form can be written for $E_{R}$ of the right boundary. The maximization of a form like (15) can be attained by a standard 1-dimensional dynamic programming procedure.

In the presence of inter-curve constraints, however, the two boundaries must be considered collectively. If we define

$$
\begin{gathered}
X_{L R, n}=\left(x_{L, n}, x_{R, n}\right) \\
S_{G}^{\prime}\left(X_{L R, n}, X_{L R, n+1}\right)=S_{G}\left(x_{L, n}, x_{L, n+1}\right)+S_{G}\left(x_{R, n}, x_{R, n+1}\right)
\end{gathered}
$$


equation (13) can be arranged in a similar form to (15) and thus be maximized by the following recursive equations:

$$
\begin{array}{rlr}
E_{2}^{\prime}\left(X_{L R, 2}\right) & = & \max _{X_{L R, 1}} S_{G}^{\prime}\left(X_{L R, 1}, X_{L R, 2}\right) \\
E_{n+1}^{\prime}\left(X_{L R, n+1}\right) & =\max _{X_{L, n}}\left(S_{G}^{\prime}\left(X_{L R, n}, X_{L R, n+1}\right)+E_{n}^{\prime}\left(X_{L R, n}\right)\right) \\
& n=2, \ldots, N-1 \\
\max _{X_{L, 1}, X_{L, 2}, \ldots, X_{L, N}} E & = & \max _{X_{L R, N}}\left(E_{N}^{\prime}\left(X_{L R, N}\right)\right)
\end{array}
$$

This procedure is called forward recursion in this paper. We can think of it as the integration of two 1D DP's (thus the name dual-DP). The optimal path is obtained by back-tracing the maximum from the last cut-line.

In the recursive computation of the local optimal path for each point on cut-line $n+1$ (refer to (19)), inter- and intra-curve constraints are enforced at the same time. For the orientation constraints (1), size constraints (7), and hard constraints (8), the variables $X_{L R, n+1}$ and $X_{L R, n}$ can be used directly to check whether these constraints are satisfied. If either of them is not, the path to $X_{L R, n+1}$ is made invalid. To exert the angular constraints $(2) \sim(\underline{6})$, backtracing is needed. For angular constraints of the $k$-th order it is necessary to trace back $k$ steps from the maximum at $X_{L R, n}$. The locally traced segments are then checked to see whether the required constraints are satisfied. It is to note that in the conventional DP methods, back-tracing is used only in finding the final path when the last step of forward recursion has completed. In our approach, back-tracing is mixed with forward recursion. The computational cost of the extra backward tracing is negligible when compared with that of forward recursion, whereas in conventional DP methods the use of high order constraints will cause an exponential increase in the computational cost [1].

\section{$3 \quad$ Experiments}

\subsection{Initialize the Search Area}

To apply the piecewise linear model to spine boundary localization, the user needs to specify the first and last cut-lines in the image plane. We do this by manually selecting the beginning and ending points on the left and right spine boundaries. This provides also hard constraints on each boundary.

\subsection{Examples}

We first demonstrate how intra- and inter-curve constraints affect the detection results. Figure 2 (a) shows an original spine image. We set an orientation threshold at 60 degrees, and an inter-segment angular threshold at 50 degrees. Figure 2(b) shows the detected boundary curves with these intra-curve constraints applied, but without inter-curve ones. It can be seen that although the detected left boundary reflects well the true position of the spine's left boundary, the right boundary has serious errors due to the presence of a spurious strong edge 


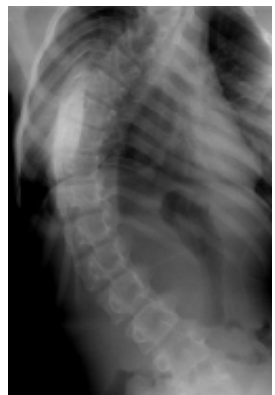

(a)

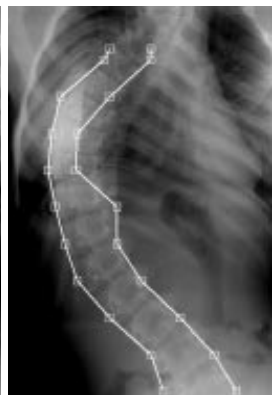

(b)

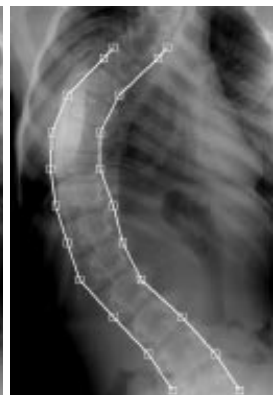

(c)

Fig. 2. The effects of inter-curve constraints. (a) the original spine image; (b) detected boundary without inter-curve constraints; (c) detected boundary by the dual-DP approach with inter-curve constraints.

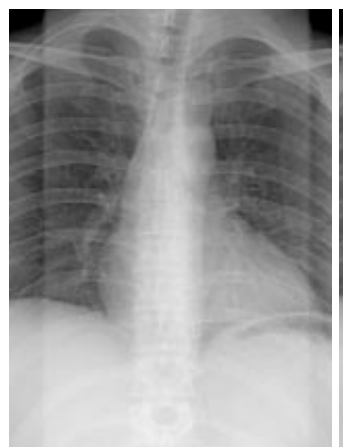

(a)

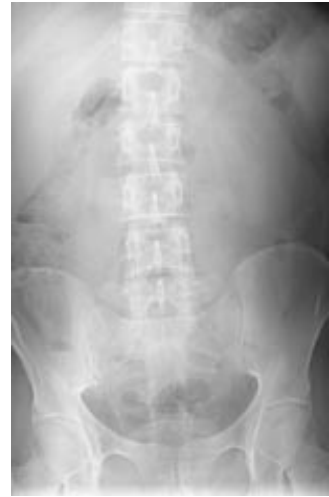

(c)

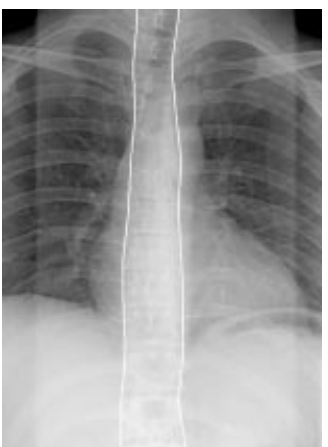

(b)

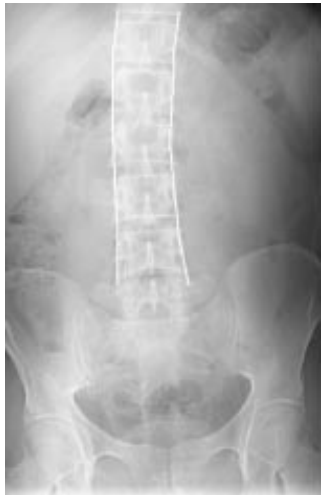

(d)

Fig. 3. Two more examples of spine boundary detection. 
in the middle of the spine. With the inter-curve constraints, we get the detected curves shown in Fig. 2(c). By comparing Figs. 2 (b) and (c), we can see that the dual-DP method not only finds the correct position for the right boundary, but also improves the localization accuracy for the left boundary.

In the above example, the image size is $370 \times 520$ pixels. It took 12 seconds on a Pentium III $500 \mathrm{MHz}$ PC to find the boundary.

Figure 3 shows two further examples of spine boundary detection by the dualDP method. Even with gradual intensity changes (Fig.3(a)), a good localization can still be obtained.

The method has been tested on a small database of 14 images. The results are qualitatively evaluated by visual inspection. Except for one case which needs manual improvement, all other images give satisfactory and good localization results.

With the detected spine boundaries as reference positions, other anatomical landmarks can be searched for and used for quantifying the deformity of a pathological spine [6].

\section{Conlusions}

In this paper, we have presented a dual dynamic programming approach to the detection of spine boundaries. As a distinctive feature, the method is able to detect coupled curves simultaneously while keeping them constrained by each other by problem-specific priors.

We proposed a piecewise linear model for the representation of boundaries. Angular measurements between segments were used to replace the conventional coordinate-based smoothness constraint. Through bound limits, it is easier to control the final shape of the detected curves. High order priors can be imposed by the mixture of forward recursion and backward tracing, without causing extra computational burdens. Experiments show that reliable results have been achieved.

\section{References}

1. A. A. Amini, T.E. Weymouth, and R.C. Jain, "Using dynamic programming for solving variational problems in vision," IEEE Trans PAMI, Vol.12, No.9, 1990, pp.855-867.

2. L.D. Cohen, "Note: on active contour models and balloons," CVGIP: Image Understanding, Vol.53, no.2, pp.211-218, 1991.

3. D. Geiger, A. Gupta, L.A. Costa, and J. Vlontzos, "Dynamic programming for detecting, tracking, and matching deformable contours", IEEE Trans PAMI, Vol.17, No.3, 1995, pp.294-402.

4. M. Kass, A. Witkin, and D. Terzopoulos, "Snakes: Active contour models," Proc. ICCV, pp.321-331, 1988.

5. R. Malladi, J.A. Sethian, and B. C. Vemuri, "Shape modeling with front propagation", IEEE Trans PAMI, Vol.17, No.2, 1995, pp.158-175.

6. B. Verdon, et. al, "Computer Assisted Quantitative Analysis of Deformities of the Human Spine", Proc. MICCAI pp.822-831, 1998. 